\title{
On the Identification of Surface Waves in Numerical Studies
}

\author{
Tom G. Mackay ${ }^{1}$ (D) \\ Received: 15 March 2018 / Accepted: 14 May 2018 / Published online: 28 May 2018 \\ (C) The Author(s) 2018
}

\begin{abstract}
In a recent study published in this journal, in which surface waves were investigated numerically for a certain equichiral thin film, the criterion used to determine whether or not surface waves were excited is not adequately discriminating.
\end{abstract}

Keywords Surface-plasmon-polariton wave · Dispersion relation - Cavity resonance

In a recent study, the prism-coupled excitation of surfaceplasmon-polariton waves (SPPWs) was investigated numerically for the interface of a metal and an equichiral thin film (ETF) [1]. Since the constitutive properties of the ETF under investigation varied periodically in the direction of the ETF's thickness, more than one SPPW could be excited at a given wavelength by changing the direction of the incident light. The criterion used to identify an SPPW was based on the magnitude of peaks in plots of absorptance versus angle of incidence: if the absorptance peak was greater than 0.4 , then this peak was claimed to represent the excitation of an SPPW. No dispersion relation from the associated canonical boundary-value problem [2] was solved in order to confirm this claim.

Generally, in studies on SPPWs, many peaks may be observed in the plots of absorptance versus angle of incidence. As is comprehensively reported in the literature [2], some of these peaks may represent SPPWs, but this needs to be confirmed by checking that the wavenumbers agree with those that arise as roots of the dispersion relation of the associated canonical boundary-value problem. Some of the absorptance peaks may arise for other reasons such as cavity resonances. These absorptance peaks should be carefully distinguished from the absorptance peaks that can be attributed to SPPWs. The wavenumbers for cavity resonances do not arise as roots of the dispersion relation for SPPWs. Furthermore, cavity resonances are highly sensitive to the thickness of the thin film in the prism-coupled

Tom G. Mackay

T.Mackay@ed.ac.uk

1 School of Mathematics and Maxwell Institute for Mathematical Sciences, University of Edinburgh, Edinburgh EH9 3FD, UK configuration, whereas SPPWs are not when the thin film's thickness is greater than a certain threshold [3, 4].

Accordingly, some of the absorptance peaks of magnitude greater than 0.4 in Ref. [1] may well be attributable to cavity resonances (and/or other mechanisms) but not to SPPWs as claimed in that paper. As a representative counterexample, many absorptance peaks of magnitude greater than 0.4 were reported in Ref. [5] but these were not attributable to SPPWs because they did not correspond to roots of the dispersion relation of the associated canonical boundary-value problem.

To summarize, the only infallible way to identify an SPPW is to solve the associated canonical boundary-value problem $[5,6]$.

In order to illustrate this matter further, the essentials of the associated canonical boundary-value problem are presented here; for comprehensive details, readers are referred elsewhere [2]. The canonical boundary-value problem is considered for electromagnetic-surface-wave propagation at the planar interface of two dissimilar materials: one occupying the half-space $z>0($ labeled $\mathcal{A})$ and the other occupying the half-space $z<0($ labeled $\mathcal{B})$. The general formalism can accommodate many different types of electromagnetic surface wave, not just SPPWs; for examples, Fano waves, Zenneck waves, Dyakonov waves, and Dyakonov-Tamm waves are also accommodated. In the interests of generality, both partnering materials may be taken to be bianisotropic and periodically nonhomogeneous in the $z$ direction [7]. Thus, the constitutive relations of these two partnering materials may be expressed as

$$
\left.\begin{array}{rl}
\mathbf{D}(\mathbf{r}) & =\underset{=}{\underline{\varepsilon}}{ }^{\mathcal{A}}(z) \cdot \mathbf{E}(\mathbf{r})+\underset{=}{\xi \mathcal{A}}(z) \cdot \mathbf{H}(\mathbf{r}) \\
\mathbf{B}(\mathbf{r}) & =\underset{=}{\zeta^{\mathcal{A}}}(z) \cdot \mathbf{E}(\mathbf{r})+\underset{\mu}{\mu}(z) \cdot \mathbf{H}(\mathbf{r})
\end{array}\right\}, \quad z>0
$$


and

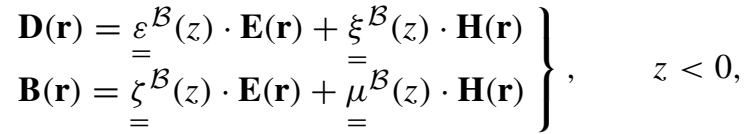

wherein $\underline{\varepsilon}^{m}(z), \underline{\xi}^{m}(z), \underline{\zeta}^{m}(z)$, and $\mu^{m}(z),(m \in\{\mathcal{A}, \mathcal{B}\})$, are $3 \times 3$ constitutive dyadics. Consequently, the formalism is not restricted to metals and dielectric partnering materials (as applies in the case for SPPWs), but non-metals and many other types of material of recent interest [8] are also accommodated. Suppose that the electromagnetic surface wave propagates in the $x y$ plane at an angle $\psi \in\left[0^{\circ}, 360^{\circ}\right]$ relative to the positive $x$ axis. The electric field phasors may then be expressed as

$\mathbf{E}(\mathbf{r})=\left\{\begin{array}{l}{\left[C_{1}^{\mathcal{A}} \mathbf{e}_{1}^{\mathcal{A}}(z)+C_{2}^{\mathcal{A}} \mathbf{e}_{2}^{\mathcal{A}}(z)\right] \exp [i q(x \cos \psi+y \sin \psi)], \quad z>0} \\ {\left[C_{3}^{\mathcal{B}} \mathbf{e}_{3}^{\mathcal{B}}(z)+C_{4}^{\mathcal{B}} \mathbf{e}_{4}^{\mathcal{B}}(z)\right] \exp [i q(x \cos \psi+y \sin \psi)], \quad z<0}\end{array}\right.$,

wherein $q$ is a complex-valued wavenumber, $C_{1,2}^{\mathcal{A}}$ and $C_{3,4}^{\mathcal{B}}$ are complex-valued amplitudes, and the two eigenvectors $\mathbf{e}_{1,2}^{\mathcal{A}}(z)$ decay as $z \rightarrow \infty$ whereas the two eigenvectors $\mathbf{e}_{3,4}^{\mathcal{B}}(z)$ decay as $z \rightarrow-\infty$. By implementing the usual boundary conditions at $z=0$ (i.e., the tangential components of the electric and magnetic field phasors must be continuous across the planar interface $z=0$ ), the following matrix-vector equation arises:

$[\underline{Y}(q)] \cdot\left[\begin{array}{c}C_{1}^{\mathcal{A}} \\ C_{2}^{\mathcal{A}} \\ C_{3}^{\mathcal{B}} \\ C_{4}^{\mathcal{B}}\end{array}\right]=\left[\begin{array}{l}0 \\ 0 \\ 0 \\ 0\end{array}\right]$

The components of the $4 \times 4$ matrix $[\underline{Y}(q)]$ depend on the constitutive dyadics of partnering materials $\mathcal{A}$ and $\mathcal{B}$, as well as on the propagation angle $\psi$. In order for a non-trivial solution to Eq. 4 to exist, $[\underline{\underline{Y}}(q)]$ must be singular, i.e.,

$\operatorname{det}\{[\underline{Y}(q)]\}=0$.

Equation 5 constitutes the dispersion relation which can be solved in order to determine the wavenumber $q$. Numerical methods are generally required to extract $q$ from Eq. 5 .

Open Access This article is distributed under the terms of the Creative Commons Attribution 4.0 International License (http:// creativecommons.org/licenses/by/4.0/), which permits unrestricted use, distribution, and reproduction in any medium, provided you give appropriate credit to the original author(s) and the source, provide a link to the Creative Commons license, and indicate if changes were made.

\section{References}

1. Hosseininezhad SH, Babaei F (2018) Excitation of multiple surface plasmon-polaritons by a metal layer inserted in an equichiral sculptured thin film. Plasmonics https://doi.org/10.1007/s11468018-0701-y

2. Polo Jr JA, Mackay TG, Lakhtakia A (2013) Electromagnetic surface waves: a modern perspective. Elsevier, Waltham

3. Motyka MA, Lakhtakia A (2008) Multiple trains of same-color surface plasmon-polaritons guided by the planar interface of a metal and a sculptured nematic thin film. J Nanophoton 2:021910

4. Motyka MA, Lakhtakia A (2008) Multiple trains of same-color surface plasmon-polaritons guided by the planar interface of a metal and a sculptured nematic thin film. Part II: Arbitrary incidence. J. Nanophoton. 3:033502

5. Polo Jr JA, Mackay TG, Lakhtakia A (2011) Mapping multiple surface-plasmon-polariton-wave modes at the interface of a metal and a chiral sculptured thin film. J Opt Soc Am B 28(11):26562666

6. Faryad M, Polo Jr JA, Lakhtakia A (2010) Multiple trains of samecolor surface plasmon-polaritons guided by the planar interface of a metal and a sculptured nematic thin film. Part IV: Canonical problem. J Nanophoton 4:043505

7. Mackay TG, Lakhtakia A (2010) Electromagnetic anisotropy and bianisotropy: a field guide. Word Scientific, Singapore

8. Tang W, Wang L, Chen X, Liu C, Yu A, Lu W (2016) Dynamic metamaterial based on the graphene split ring high-Q Fanoresonnator for sensing applications. Nanoscale 8:15196 